\title{
L’Éditeur et le Graveur en société avec le Poète : Poulet-Malassis, Félicien Rops, et Baudelaire en Belgique
}

\section{Nicolas Valazza}

Quand bien même aucun poème des Fleurs $d u$ Mal n'aurait été écrit à partir de $1863,{ }^{1}$ Baudelaire n'a pas cessé pour autant de composer des vers dans les dernières années de sa vie, jusqu'à sa chute dans l'église Saint-Loup à Namur en mars 1866, entraînant sa paralysie progressive, son aphasie, et finalement sa mort à Paris le 31 août de l'année suivante. ${ }^{2}$ L'exil volontaire du poète en Belgique dès avril 1864 est certes caractérisé par le tarissement de sa veine lyrique au profit de la verve du pamphlétaire, dont témoignent surtout les brouillons de La Belgique déshabillée $;^{3}$ mais le séjour belge de Baudelaire n'est pas moins marqué par une œuvre en vers, dont il est toutefois difficile d'estimer l'étendue, du fait que le poète ne signe pas tous ses écrits. On peut le déduire de la lettre du 31 octobre 1864 à Poulet-Malassis, où Baudelaire prie son éditeur de lui confier « un ouvrage sur lequel [il] serai[t] fier de coller son nom $» ;^{4}$ ce qui implique qu'il a contribué, dans une certaine mesure qu'il est sans doute impossible d'établir, aux ouvrages clandestins édités par Poulet-Malassis à Bruxelles. ${ }^{5}$ Baudelaire avait rejoint son éditeur exilé en Belgique pour tâcher, entre autres raisons, de s'acquitter envers lui d'une « dette » tant morale que pécuniaire, après avoir cédé les droits de ses œuvres à Pierre-Jules Hetzel en 1863, alors qu'ils appartenaient exclusivement à PouletMalassis : «J'accepte, écrit Baudelaire dans la même lettre, [...] le bizarre travail que vous m'avez proposé » (il s'agit de la traduction d'un texte licencieux en latin). « Quant au salaire, ajoute-t-il, ce que vous voudrez;- - payé comme vous voudrez,- - en argent ou en billets,—ou 
bien compté comme diminution de ma dette»(Corr 2:416). Aussi la collaboration entre l'éditeur et le poète concerne-t-elle surtout des œuvres de commande : traductions du latin, anthologies érotiques, ${ }^{6}$ éditions critiques d'œuvres libertines du XVIII ${ }^{\mathrm{e}}$ siècle, ${ }^{7}$ parodies obscènes, ${ }^{8}$ etc.

Poulet-Malassis fait cependant paraître, en février ou mars 1866, l'édition clandestine des Épaves de Charles Baudelaire, ${ }^{9}$ un ouvrage dont le poète supervise tout le processus éditorial, depuis la composition du recueil jusqu'à la diffusion du livre, malgré l'«Avertissement de l'éditeur » qui prétend que «l'auteur sera avisé de cette publication en même temps que les deux cent soixante lecteurs probables » $\left(O C\right.$ 1:812). ${ }^{10}$ Les Épaves ne sont pas moins le fruit de la collaboration entre le poète et l'éditeur, auxquels il faut ajouter l'aquafortiste belge Félicien Rops. Le recueil de poèmes est en effet précédé d'une « eauforte frontispice » dessinée et gravée par Rops, que Baudelaire considère comme «le seul véritable artiste » qu'il ait « trouvé en Belgique ». ${ }^{11}$ De fait, une solide amitié en vient à lier l'éditeur, le graveur et le poète, en dépit du prétendu « isolement » dont ce dernier ne cesse de se plaindre au cours de son séjour belge $;^{12}$ une amitié qui s'accompagne donc d'une collaboration active à plusieurs projets d'éditions. ${ }^{13}$ En ce qui concerne Les Épaves, ce recueil se distingue par le point de vue rétrospectif qu'il présente sur Les Fleurs du Mal, en assumant tout à la fois : une fonction complémentaire, en ajoutant des «nouvelles Fleurs »; $;^{14}$ une fonction rédemptrice, en rééditant les six «pièces condamnées » de l'édition de 1857 '15 $^{15}$ une fonction exégétique, en interprétant le «Mal »dans un sens résolument peccamineux; et enfin une fonction visuelle, au moyen de l'eau-forte de Rops [Fig. 1]. 


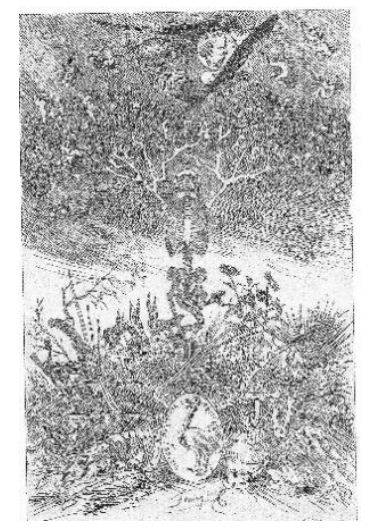

Figure 1 : Félicien Rops, frontispice pour Les Épaves de Charles Baudelaire (Amsterdam : À l'enseigne du Coq [Bruxelles : Poulet-Malassis], 1866). Reproduit avec l'autorisation du Kinsey Institute, Indiana University Bloomington.

Ce frontispice était destiné à l'ensemble des Fleurs $d u M a l,{ }^{16}$ et sa conception dans l'esprit de Baudelaire précède de fait la deuxième édition du recueil, comme le prouve une lettre d'août 1860 à Poulet-Malassis : «Un squelette arborescent, l'arbre de la science du bien et du mal, à l'ombre duquel fleurissent les sept péchés capitaux sous la forme de plantes allégoriques » (Corr 2:87). Ce projet avait d'abord été commandé à Félix Bracquemond, qui n'avait toutefois pas su satisfaire le poète (pour user d'un euphémisme ${ }^{17}$ ); aussi est-ce en Félicien Rops que Baudelaire allait trouver un talent apte à matérialiser son imaginaire symbolique. ${ }^{18}$ Le poète et le graveur partagent en effet un même goût pour le détournement de l'iconographie chrétienne, dans une exaltation du péché, fatalement suivi de remords dans le cas de Baudelaire, avec une insouciance païenne dans le cas de Rops. ${ }^{19}$ En l'occurrence, l'image détournée est celle d'une gravure du XVI siècle de Sebald Beham [Fig. 2] qui représente précisément « l'arbre de science du bien et du mal » sous les traits d'un « squelette arborescent $\gg .^{20}$ 


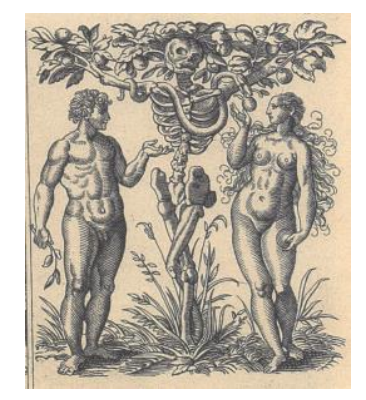

Figure 2: Sebald Beham, Adam et Ève ( $\mathrm{XVI}^{\mathrm{e}}$ siècle), dans Eustache-Hyacinthe Langlois, Essais historique, philosophique et pittoresque sur les danses des morts, vol. 2 (Rouen: A. Lebrument, 1851), pl. VII.

La puissance de synthèse du dessin de Rops parvient cependant à condenser les éléments naturels (les «plantes »), théologiques (les «Sept Péchés Capitaux»), érotiques (le «Serpent» phallus), mythologiques (le «Pégase macabre », la «Chimère noire ») et emblématiques (l'«Autruche en camée, [...] emblème de la Vertu ») de l'image ; une puissance visuelle qui concrétise de la sorte une pensée allégorique, et qui trouve son équivalent textuel dans la poésie de Baudelaire. ${ }^{21}$

Le volume des Épaves constitue donc bien un compendium iconotextuel des Fleurs du Mal, tout en historicisant l'œuvre de Baudelaire près de dix ans après la parution de la première édition du recueil. Tel est le propos du sonnet liminaire des Épaves, « Le Coucher du soleil romantique », composé en $1862:^{22}$

Que le Soleil est beau quand tout frais il se lève,

Comme une explosion nous lançant son bonjour !

—Bienheureux celui-là qui peut avec amour

Saluer son coucher plus glorieux qu'un rêve !

Je me souviens !... J'ai vu tout, fleur, source, sillon, 
Se pâmer sous son œil comme un cœur qui palpite...

-Courons vers l'horizon, il est tard, courons vite,

Pour attraper au moins un oblique rayon!

Mais je poursuis en vain le Dieu qui se retire ;

L’irrésistible Nuit établit son empire,

Noire, humide, funeste et pleine de frisson ;

Une odeur de tombeau dans les ténèbres nage,

Et mon pied peureux froisse, au bord du marécage,

Des crapauds imprévus et de froids limaçons. (OC 1:149)

La tonalité crépusculaire de ce sonnet l'assimile à un «épilogue » ${ }^{23}$ plutôt qu'à un poème liminaire, en le rapprochant des dernières strophes du «Voyage » qui conclut Les Fleurs du Mal : «Si le ciel et la mer sont noirs comme de l'encre, / Nos cœurs que tu connais [ô Mort] sont remplis de rayons!» (OC 1:134). Or Les Fleurs du Mal, cette floraison ultime de la poésie romantique, si ce n'est ultérieure à la poésie romantique, sont bien achevées en 1866, même si elles n'ont pas encore toutes paru en volume; de sorte que « Le Coucher de soleil romantique » ne pouvait coïncider, pour Baudelaire, qu'avec l'épuisement de la poésie et de l'art, étant établi, depuis le «Salon de 1846 », que «qui dit romantisme dit art moderne, c'est-à-dire intimité, spiritualité, couleur, aspiration vers l'infini, exprimées par tous les moyens que contiennent les arts » $(O C$ 2:421), y compris la poésie. «L’irrésistible Nuit [qui] établit son empire » plonge donc dans les ténèbres toute œuvre poétique : non seulement celle du sujet lyrique («je poursuis en vain le Dieu qui se retire»), mais aussi celle de sa 
génération, ${ }^{24}$ voire la poésie lyrique en général (Baudelaire ne pouvait certes pas prévoir le rôle de précurseur qui allait lui être attribué par les poètes parnassiens puis symbolistes ${ }^{25}$ ).

Cette sombre vision de la poésie trouve certes son emblème dans le squelette de Pégase, le symbole des poètes échoué parmi les fleurs maléfiques dans le frontispice de Rops ; ce même Pégase qui, dans le prologue des Chansons des rues et des bois de Hugo, publiées l'année précédente à Bruxelles, « souffle l'ode, l'épopée / Le drame », et fait « Jaillir pour les Grecs Hippocrène », ${ }^{26}$ à savoir la source des Muses — et par là même de la poésie lyrique, épique et dramatique - sur le mont Hélicon. ${ }^{27} \mathrm{Ce}$ «Pégase macabre » allait du reste reparaître dans l'« ex-libris » gravé par Rops pour la première édition des Poésies de Mallarmé en $1887,{ }^{28}$ et de nouveau dans l'édition posthume et définitive de 1899: preuve s'il en fallait que le lyrisme ne cesse d'expirer, et partant de renaître de ses cendres tel un phénix, dans les dernières décennies du XIX ${ }^{\mathrm{e}}$ siècle.

C'est pourtant à un autre frontispice [Fig. 3], dessiné par Rops pour une réédition clandestine des Jeunes-France de Théophile Gautier par les soins de Poulet-Malassis en 1866, que «Le Coucher du soleil romantique » versifié par Baudelaire paraît plus étroitement lié. 


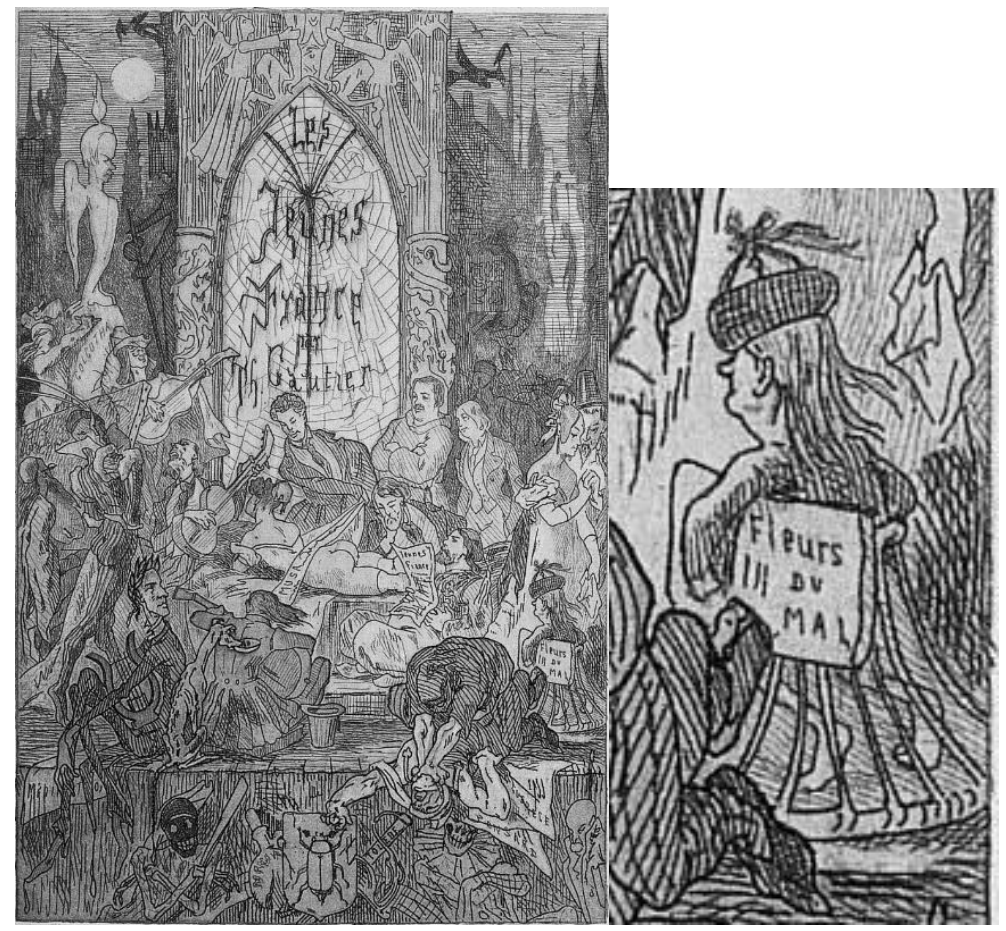

Figure 3 : Félicien Rops, frontispice pour Théophile Gautier, Les Jeunes-France. Romans goguenards, 1833 (Amsterdam : À l'enseigne du Coq [Bruxelles : Poulet-Malassis], 1866).

Rops décrit son propre dessin et énumère les personnages qui y figurent dans une lettre non référencée :

Alexandre Dumas jeune découvre la Muse qu'Alfred de Musset regarde de trop près. Balzac en moine, George Sand en homme : M. de Girardin ; Théophile Gautier en Turc, Sainte-Beuve, Lamartine, toute l'école romantique fait galerie. Dans le fond, Hugo «Golgothe ». Tous ces personnages sont jeunes. À l'avant-plan, Ponsard en Romain est étranglé par un Pétrus Borel quelconque. Le dernier venu, Baudelaire, en bourrelet, apporte ses Fleurs du Mal. ${ }^{29}$

Ce panthéon romantique, qui était à son apogée dans les années 1830, semble bien déchu en 1866, du fait que la plupart de ses divinités-ou semi-divinités—sont soit mortes (Musset, 
Vigny, Balzac, Borel), soit près de s'éteindre (Dumas, Sainte-Beuve, Lamartine, Ponsard), soit exilées (Hugo), soit elles ont renoncé à la ferveur romantique de leur jeunesse (Gautier) ; autrement dit, le romantisme fait désormais partie de l'histoire, telle qu'allait l'écrire Gautier dans sa dernière œuvre inachevée. ${ }^{30}$ Aussi Baudelaire, « le dernier venu », appartient-il à une autre génération que celle des «mages romantiques » et de leurs avatars, quoique sa place dans l'histoire littéraire ne soit pas encore assignée, pas plus que ne l'est celle des Fleurs du Mal : il est le poète de l'après-coup, celui qui est arrivé trop tard, en dégageant un sentiment d'inactualité qu'allaient éprouver nombre de poètes de la fin du siècle, et par-dessus tous Mallarmé. ${ }^{31}$

Le panthéon romantique représenté par Rops paraît toutefois peu conventionnel, dans la mesure où «la Muse » étendue au centre de l'image est dépeinte sous les traits d'une courtisane dévêtue, coiffée à la mode du siècle et exhibant son postérieur ; une muse qui ne saurait donc correspondre à La Muse française désignant, dès 1823, la revue et le premier cénacle des poètes romantiques (Soumet, Guiraud, Deschamps, Hugo, Sophie Gay, Vigny, parmi d'autres), unis dans le culte d'un lyrisme aussi chaste que religieux..$^{32}$ Plusieurs poètes figurant dans le frontispice n'avaient certes pas hésité à cultiver une muse plus lascive, telle celle qu'invoquent les vers attribués à Musset dans Le Nouveau Parnasse satyrique : «Ce qu'il me faut, à moi, c'est la brutale orgie, / La brune courtisane à la lèvre rougie, / Qui se pâme et se tord » (76). ${ }^{33}$ Mais le portrait de «la Muse » dessiné par Rops se réfère sans doute aussi à «La Muse vénale » de Baudelaire :

Ô muse de mon cœur, amante des palais, Auras-tu, quand Janvier lâchera ses Borées, Durant les noirs ennuis des neigeuses soirées, Un tison pour chauffer tes deux pieds violets? 
Ranimeras-tu donc tes épaules marbrées

Aux nocturnes rayons qui percent les volets ?

Sentant ta bourse à sec autant que ton palais,

Récolteras-tu l'or des voûtes azurées ?

Il te faut, pour gagner ton pain de chaque soir,

Comme un enfant de chœur, jouer de l'encensoir,

Chanter des Te Deum auxquels tu ne crois guère,

Ou, saltimbanque à jeun, étaler tes appas

Et ton rire trempé de pleurs qu'on ne voit pas,

Pour faire épanouir la rate du vulgaire. (OC 1:15)

Ce sonnet, publié dans la première édition des Fleurs du Mal en 1857, est fort antérieur aux Épaves; mais il ne décrit pas moins fidèlement la situation de Baudelaire au cours de son exil volontaire en Belgique, qui le voit réduit à composer et à éditer sur commande des ouvrages licencieux pour le compte de Poulet-Malassis. En ce sens, le poème procède à l'incorporation de « la muse vénale » à la personne du poète (qui est d'ailleurs travesti en courtisane dans le dessin de Rops), lui-même figuré par le «saltimbanque à jeun »contraint, «pour gagner [s]on pain de chaque soir », de « faire épanouir la rate ${ }^{34}$ du vulgaire », c'est-à-dire de divertir le bourgeois. ${ }^{35}$

On peut douter, cependant, que Baudelaire se soit adonné à contrecœur à l'écriture grivoise, quoi qu'il en dise dans sa correspondance. ${ }^{36} \mathrm{Au}$ demeurant, Les Épaves se distinguent par une plus grande liberté de mœurs que celle des Fleurs du Mal, en reprenant 
les «Pièces condamnées » et en proposant de nouvelles «Galanteries », écrites en Belgique, dont l'érotisme est tantôt mesuré, comme dans « Les Promesses d'un visage » :

Tu trouveras au bout de deux beaux seins bien lourds,

Deux larges médailles de bronze,

Et sous un ventre uni, doux comme du velours,

Bistré comme la peau d'un bonze,

Une riche toison qui, vraiment, est la sœur

De cette énorme chevelure,

Souple et frisée, et qui t'égale en épaisseur,

Nuit sans étoiles, Nuit obscure ! (OC 1:163)

Tantôt il débouche franchement sur l'obscène, comme dans «Le Monstre, ou le Paranymphe d'une nymphe macabre » $: 37$

Oh ! très sincèrement je souffre

De ne pas aller aux sabbats,

Pour voir, quand il [le Diable] pète du soufre,

Comment tu lui baises son cas ! $!^{38}$

Oh ! très sincèrement je souffre ! $(O C 1: 166)$

Baudelaire avait envoyé ces «Galanteries » à Catulle Mendès en janvier 1866 pour qu'il les fît paraître dans Le Parnasse contemporain $;^{39}$ ce que celui-ci refusa, en partie, de faire en 
arguant que «quelques pièces, trop vives, ne peuvent prendre place dans le Parnasse ${ }^{40}$ Mendès se réfère précisément aux «Promesses d'un visage » et au «Monstre » qui, en effet, ne font pas partie des « Nouvelles Fleurs $d u$ Mal» parues dans la cinquième livraison du Parnasse le 31 mars, ${ }^{41}$ alors que Baudelaire était atteint d'hémiplégie à la suite de sa chute à Namur quinze jours plus tôt, et qu'il avait définitivement cessé d'écrire. Dans «Le Monstre », l'un des tout derniers poèmes composés par Baudelaire, celui-ci renoue donc avec la veine charnelle, littéralement sulfureuse («il pète du soufre ») et maléfique, qui avait entraîné la condamnation de six poèmes des Fleurs $d u$ Mal en 1857 :

\author{
Voulant du Mal chercher la crème \\ Et n'aimer qu'un monstre parfait, \\ Vraiment oui ! vieux monstre, je t'aime ! (OC 1:166)
}

Cette ultime «fleur», ou plutôt cette «épave» pour l'heure impubliable, du moins légalement, ${ }^{42}$ se rapproche de certaines «pièces condamnées » également par son caractère dédicatoire et circonstanciel. De même que les vers d' « À celle qui est trop gaie » avaient été adressés, anonymement, par Baudelaire à Apollonie Sabatier le 9 décembre 1852 (Corr 1:205-6), ainsi le poème du « Monstre » est dédié, d'après le manuscrit, «à Madame B... », ${ }^{43}$ dont l'identité est restée inconnue, en dépit des nombreuses hypothèses avancées par les commentateurs. $^{44}$ Pareillement, le manuscrit des «Promesses d'un visage » révèle une dédicace, antérieure au titre, «à Mademoiselle A...z », tout aussi inconnue, s'il ne s'agit de Berthe, la «dernière maîtresse » supposée de Baudelaire, invoquée dans une autre «galanterie » intitulée précisément «Les Yeux de Berthe » $\left(O C\right.$ 1:166). ${ }^{45}$ Quoi qu'il en soit, les derniers vers de Baudelaire se distinguent par la présence dominante de leurs destinataires, qui en viennent à reléguer le sujet lyrique, l'énonciateur des poèmes, au second 
rôle, résolument mineur par rapport à la subjectivité poussée à l'extrême des premières Fleurs $d u$ Mal, notamment de la section « Spleen et Idéal ».

On le constate, sur un autre registre, dans le sonnet de circonstance adressé par Baudelaire à Poulet-Malassis en avril 1865 «pour s'excuser de ne pas accompagner un ami à Namur » :

Puisque vous allez vers la ville

Qui bien qu'un fort mur l'encastrât,

Défraya la verve servile

Du fameux poète Castrat,

Puisque vous allez en vacances

Goûter un plaisir recherché,

Usez toutes vos éloquences,

Mon bien cher Coco-Malperché

(Comme je le ferais moi-même)

À dire là-bas combien j'aime

Ce tant folâtre Monsieur Rops

Qui n'est pas un grand prix de Rome,

Mais dont le talent est haut comme

La pyramide de Chéops. (Corr 2:485-86)

Ce sonnet a souvent été exclu, au même titre que d'autres vers de circonstances, ${ }^{46}$ des « œuvres [soi-disant] complètes » de Baudelaire, ${ }^{47}$ alors même qu'il est emblématique de la 
dernière manière du poète, fortement marquée par la présence de l'éditeur et du graveur, ses deux «amis » en terre belge. Les tercets constituent ainsi un hommage sincère à la qualité d'illustrateur (qui n'est donc pas celle d'« un grand prix de Rome », c'est-à-dire d'un peintre académique) de Rops, dont le nom est accouplé, par une rime des plus rares, si ce n'est unique (plus rare en tout cas que la rime en $-y x$ de Mallarmé), à « La pyramide de Chéops », à l'aune de laquelle est mesuré le «talent» de l'artiste. Si Baudelaire fait montre d'une inspiration poétique de plus en plus intermittente au cours de son exil en Belgique, il n'a pas perdu pour autant sa puissance de figuration; de sorte qu'en dénonçant, dans le premier quatrain, «la verve servile / Du fameux poète Castrat », Baudelaire sous-entend qu'il n'est pas lui-même atteint d'impuissance poétique. D'après une légende colportée depuis le $\mathrm{XVII}^{\mathrm{e}}$ siècle, ce « poète Castrat » doit être identifié à Nicolas Boileau, ${ }^{48}$ qui avait composé une «Ode sur la prise de Namur» en l'honneur de Louis XIV après sa conquête-très provisoire-de la ville «encastr[ée]» en 1692; Namur qui est précisément le lieu de naissance et de résidence de Rops, où Baudelaire devait se rendre avec Poulet-Malassis en avril 1865. Celui-ci avait édité, l'année précédente, une «parodie [obscène] des deux premiers chants de L'Art poétique» de Boileau, intitulée L'Art priapique et illustrée plaisamment par Rops. ${ }^{49}$ Or on suppose que cette parodie, prétendument écrite par «un octogénaire » et publiée à «Namur, à l'enseigne de Boileau dindonné » (le frontispice de Rops montre un dindon qui s'apprête à émasculer Boileau enfant ${ }^{50}$ ), a pu être composée par Baudelaire, ou éventuellement en collaboration. ${ }^{51}$ À cet égard, le sonnet adressé à PouletMalassis, qui évoque plusieurs éléments paratextuels de L’Art priapique, pourrait s'apparenter à une signature cryptée de la parodie.

Force est de constater que de nombreuses zones d'ombre subsistent sur la production poétique de Baudelaire en Belgique. Du moins peut-on conclure que la licence, la circonstance, la dissimulation, la «bouffonnerie ${ }^{52}$ la récriture ludique et, d'une manière 
générale, la collaboration y assument une fonction primordiale. En ce sens, la poésie serait devenue, dans les derniers vers de Baudelaire, le lieu d'une communication, notamment avec les amis et les amantes, d'autant plus intime qu'elle pouvait se passer des conventions prosaïques de la correspondance régulière ; une communication qui est donc aussi celle du sujet lyrique avec lui-même, au-delà du solipsisme propre à une certaine conception romantique du lyrisme qui informe les premières Fleurs du Mal. En s'opposant, par ailleurs, au soliloque hargneux de la prose sur la Belgique, ainsi qu'aux Amonitates Belgicae (éd. Guyaux 455-66) qui en sont le complément en vers, les derniers poèmes dédicatoires de Baudelaire nous amènent dès lors à éclaircir l'image sombre, qu'il s'est lui-même plu à véhiculer, du poète exilé et isolé, dont le fiel pamphlétaire aurait entièrement desséché la veine poétique, peu avant l'aphasie définitive.

\section{Indiana University Bloomington}

${ }^{1}$ Parmi les poèmes ajoutés à la troisième édition—posthume—du recueil en 1868 , ceux qui, selon Claude Pichois, devaient effectivement faire partie du volume projeté par Baudelaire, et dont la date de composition est incertaine, sont tous antérieurs à l'année 1863 (voir $O C$ $1: 135-45)$.

${ }^{2}$ Voir le témoignage indirect d'Eugène Crépet, « Étude biographique » [1887], dans Eugène et Jacques Crépet, Charles Baudelaire (Paris : A. Messein, 1906), 187-88.

${ }^{3}$ Titre retenu par André Guyaux dans son édition du pamphlet inachevé de Baudelaire sur la Belgique, mieux connu sous le titre de Pauvre Belgique. Voir Baudelaire, Fusées. Mon cœur mis à nu. La Belgique déshabillée, éd. André Guyaux (Paris : Gallimard, 1986).

${ }^{4}$ Baudelaire, Correspondance, vol. 2, éd. Claude Pichois (Paris : Gallimard, 1973), 416 (dorénavant Corr). 
${ }^{5}$ Comme je le propose dans Nicolas Valazza, «Baudelaire au Parnasse satyrique », L'Année Baudelaire 15 (2011) : 87-101.

${ }^{6}$ Par exemple Le Parnasse satyrique du dix-neuvième siècle. Recueil de vers piquants et gaillards, 2 vols. (Rome : À l'enseigne des sept péchés capitaux, s.d. [Bruxelles, PouletMalassis, 1864]) et Le Nouveau Parnasse satyrique du dix-neuvième siècle (Eleutheropolis : Aux devantures des libraires, ailleurs, dans leurs arrière-boutiques [Bruxelles : PouletMalassis], 1866).

${ }^{7}$ Dans sa lettre du 30 mars 1865 à Sainte-Beuve, Baudelaire mentionne « quelques livres badins » (de Boyer d'Argens, d'Andréa de Nerciat et de Vivant Denon, parmi d'autres auteurs libertins du XVIII ${ }^{\mathrm{e}}$ siècle) dont il a «tiré un petit, petit bénéfice inattendu, c'est une intelligence plus nette de la Révolution française » (Corr 2:491). On sait par ailleurs que Baudelaire avait entrepris un «travail critique » sur Les Liaisons dangereuses de Laclos. Voir les « Notes sur les Liaisons dangereuses » dans Baudelaire, Fusées. Mon Cour mis à nu. Et autres fragments posthumes, éd. André Guyaux (Paris : Gallimard, 2016), 151-70. ${ }^{8}$ Éventuellement L'Art priapique. Parodie des deux premiers chants de l'Art poétique, par un Octogénaire (Namur : À l’enseigne de Boileau dindonné [Bruxelles : Poulet-Malassis], 1864).

${ }^{9}$ Les Épaves de Charles Baudelaire, avec une eau-forte frontispice de Félicien Rops (Amsterdam : À l'enseigne du coq [Bruxelles : Poulet-Malassis], 1866). Un exemplaire conservé à la Bibliothèque nationale de France est consultable en ligne : http://gallica.bnf.fr/ark:/12148/bpt6k1054576n. Consulté le 8 décembre 2017. ${ }^{10}$ En retournant les épreuves corrigées des Épaves à Poulet-Malassis, Baudelaire note sur un billet : «Mon cher ami, voici les placards, assez bien corrigés, je crois. Maintenant, il faut un en-tête, de vous, en prose, que vous signerez d'une signature quelconque (pas la vôtre) ou que 
vous ne signerez pas du tout, ou que vous signerez : l'Éditeur.-Ayez soin d'indiquer que ce recueil est fait sans la participation de l'auteur » $(O C$ 1:1120).

${ }^{11}$ Lettre du 11 mai 1865 à Édouard Manet (Corr 2:496). Dans son ébauche de pamphlet sur la Belgique, Baudelaire écrit : «En Belgique, pas d'art. Il s'est retiré du pays.—Pas d'artiste—excepté Rops, —et Leys » (éd. Guyaux 250).

${ }^{12}$ Baudelaire écrit ainsi à sa mère le 31 juillet 1864 : «Si tu savais quelle colère on éprouve quand on est complètement isolé, enfermé dans un milieu hostile, sans conversations, sans aucun plaisir possible, et quand personne de ceux de qui vous avez besoin ne vous répond ! » (Corr 2:392). Et le 30 novembre de l'année suivante à Narcisse Ancelle : « Je m’ennuie et je souffre le martyre. J'ai rompu toute espèce de relations. J'aime encore mieux une solitude absolue que les compagnies brutales, bêtes, et ignorantes » (Corr 2:548).

${ }^{13}$ Jacques Odry a recensé trente-quatre ouvrages illustrés par Rops et édités par PouletMalassis à Bruxelles entre 1864 et 1871, l'année du retour de celui-ci en France, dans «Les éditions bruxelloises de Poulet-Malassis ornées d'un frontispice de Félicien Rops », Le Livre et l'Estampe 18 (1972) : 45-75. Baudelaire a donc contribué à un certain nombre de ces éditions, quoiqu'on n'en sache le nombre et exactement dans quelle mesure.

${ }^{14}$ Dont certaines paraissent simultanément—et légalement—sous le titre de « Nouvelles Fleurs du Mal » dans Le Parnasse contemporain. Recueil de vers nouveaux, vol. 1 (Paris : A. Lemerre, 1866), 65-80, http://gallica.bnf.fr/ark:/12148/bpt6k199820. Consulté le 8 décembre 2017. ${ }^{15}$ Après qu'elles avaient reparu dans Le Parnasse satyrique (2:18-29). ${ }^{16}$ Voir le dessin préparatoire annoté par Rops à l'adresse de Poulet-Malassis : « Il est convenu n'est-ce pas comme vous me l'avez dit que nous mettons Fleurs du Mal, ce frontispice s'adressant à l'œuvre en général.—Du reste il en sera ce que vous voudrez ». Reproduit dans le catalogue de la vente de « 100 livres, manuscrits, documents et objets de la collection de Pierre Leroy » (Paris : Sotheby's, 27 juin 2007), lot 33, 
http://www.sothebys.com/fr/auctions/ecatalogue/lot.33.html/2007/100-books-manuscripts-documents-andobjects-from-the-pierre-leroy-collection-pf7025\#. Consulté le 8 décembre 2017.

17 «Voici l'horreur de Bracquemond », s'exclame Baudelaire en tête d'une lettre d'août 1860 à Poulet-Malassis (Corr 2:83).

${ }^{18}$ Sur la genèse du frontispice, voir l'étude d'Hélène Védrine, « Le frontispice des Épaves de Charles Baudelaire par Félicien Rops », dans Autour des Épaves de Charles Baudelaire, catalogue de l'exposition du musée provincial Félicien Rops de Namur, 8 mai-30 août 1999 (Anvers : Pandora, 1999), 25-50.

${ }^{19}$ Dans la même lettre à Poulet-Malassis, Baudelaire affirme que « toute littérature dérive du péché.-Je parle très sérieusement » (Corr 2:85). On se souvient, en outre, du premier quatrain du poème liminaire «Au lecteur » des Fleurs du Mal : «La sottise, l'erreur, le péché, la lésine, / Occupent nos esprits et travaillent nos corps, / Et nous alimentons nos aimables remords, / Comme les mendiants nourrissent leur vermine » (OC 1:5).

${ }^{20}$ Baudelaire avait découvert cette gravure « en feuilletant l'histoire des Danses macabres, d'Hyacinthe Langlois », comme il l'écrit à Nadar le 16 mai 1859 (Corr 1:577).

${ }^{21}$ Tous ces éléments sont mentionnés dans l'« Explication du frontispice » écrite par Poulet en regard de l'eau-forte dans le volume des Épaves (OC 1:812-13). Dans la première phase de conception du dessin, Rops fournit à Poulet-Malassis la liste des «plantes allégoriques » réclamées par Baudelaire dès 1860, figurant chacune un des sept péchés capitaux : «Iraune cactée du Cap—à longs dards / Pigritia —une souche desséchée et couchée / Invidia — une serpentaire à aiguillon / Gula — un melon / Libido — satyrion ou un arum (vit de prêtre) / Superbia—le soleil [des tournesols] / Avaritia — plantes à griffes ». Lettre non datée, citée par Claude Pichois et François Ruchon, Iconographie de Charles Baudelaire (Genève : P. Cailler, 1960), 117. 
${ }^{22}$ Comme le précise la « note de l'éditeur » (en partie, si ce n'est intégralement écrite par Baudelaire) ajoutée au poème : «Ce sonnet a été composé en 1862, pour servir d'épilogue à un livre de M. Charles Asselineau, qui n’a pas paru : Mélanges tirés d'une petite bibliothèque romantique; lequel devait avoir pour prologue un sonnet de $\mathrm{M}$. Théodore de Banville : Le Lever du soleil romantique » (OC 1:149). Les Mélanges d'Asselineau allaient de fait paraître en décembre 1866. Voir la note ci-dessous.

${ }^{23}$ Le poème paraît ainsi, sous le titre « Soleil couché », en tant que « Sonnet-Épilogue » à l'issue de l'essai bibliographique de Charles Asselineau, Mélanges tirés d'une petite bibliothèque romantique (Paris : R. Pincebourde, 1866), 149.

${ }^{24} \mathrm{La}$ « note de l'éditeur » qui accompagne ce sonnet dans Les Épaves explique que «par l'irrésistible Nuit M. Charles Baudelaire a voulu caractériser l'état actuel de la littérature, et que les crapauds imprévus et les froids limaçons sont les écrivains qui ne sont pas de son école » $(O C$ 1:149).

${ }^{25}$ En réponse à la demande de contribution au Parnasse contemporain que lui avait adressée Catulle Mendès, Baudelaire lui écrivit le 19 janvier 1866 : «Je suis assez curieux de savoir quel est le mortel audacieux, absurde, inepte, anti-progressiste et divin, qui a osé penser qu'il y aurait des lecteurs à qui des vers, même excellents, je les suppose excellents, pourraient plaire. Ainsi l'absurde est encore de ce monde. Je croyais qu'il n'y avait plus que moi dans ce genre-là » (Corr 2:574). L'année précédente, Mallarmé et Verlaine avaient publié des articles élogieux sur Baudelaire, respectivement dans L'Artiste du $1^{\text {er }}$ février et dans L'Art des 16 et 30 novembre et du 23 décembre 1865 ; si bien que le poète allait s'interroger, dans la lettre du 5 mars 1866 à sa mère, sur l'existence de «l'école Baudelaire »(Corr 2:625).

${ }^{26}$ Victor Hugo, «Le Cheval », dans Les Chansons des rues et des bois (Paris : Librairie internationale et Bruxelles : A. Lacroix, Verboeckhoven et $\left.C^{\text {ie }}, 1865\right)$, X. Baudelaire n'avait pas répondu à Hugo après que celui-ci lui eut envoyé un exemplaire dédicacé du recueil ; un 
silence qui ne manqua pas de troubler l'exilé de Guernesey, lequel en demanda raison à son fils François-Victor le 14 novembre 1865 : « D'où vient le silence [...] de

M. Ch. Baudelaire ?» (Corr 2:936).

${ }^{27}$ Voir Ovide, Les Métamorphoses, livre 5, v. 250-68.

${ }^{28}$ Les Poésies de Stéphane Mallarmé photolithographiées du manuscrit (Paris : Éditions de la Revue Indépendante, 1887), f. 3r, http://gallica.bnf.fr/ark:/12148/btv1b86268385/f13.image. Consulté le 8 décembre 2017.

${ }^{29}$ Lettre citée, entre autres, par Joséphin Peladan, « Les Maîtres contemporains : Félicien Rops », La Jeune Belgique 4 (1884-85) : 78

${ }^{30}$ Théophile Gautier, Histoire du romantisme (Paris : Charpentier, 1874).

${ }^{31}$ Mallarmé écrit notamment à Verlaine le 16 novembre 1885 : «Au fond je considère l'époque contemporaine comme un interrègne pour le poëte, qui n'a point à s'y mêler : elle est trop en désuétude et en effervescence préparatoire, pour qu'il ait autre chose à faire qu'à travailler avec mystère en vue de plus tard ou de jamais ». Stéphane Mallarmé, Euvres complètes, vol. 1, éd. Bertrand Marchal (Paris : Gallimard, « Bibliothèque de la Pléiade », 1998), 789.

${ }^{32}$ L'Avant-propos du premier numéro de La Muse française affirme ainsi : « L'amour de la poésie, comme tout autre amour, pourrait bien languir et s'éteindre faute d'alimens $[\mathrm{sic}] \mathrm{La}$ Muse française est instituée principalement pour rallumer et entretenir ce feu sacré. » $L a$ Muse française (1823-24), vol. 1, éd. Jules Marsan (Paris : É. Cornély et C $\left.{ }^{\mathrm{ie}}, 1907-1909\right), 4$. ${ }^{33}$ On peut également mentionner les six poèmes érotiques de Gautier parus anonymement dans Le Parnasse satyrique, 1:136-42.

${ }^{34}$ Spleen en anglais. Selon Émile Littré, l'expression familière « épanouir la rate » signifie « divertir, faire rire ». Dictionnaire de la langue française, 4 vols. (Paris : Hachette, 18731874), 4:1485. 
Le poète s'identifie au « Vieux Saltimbanque » dans Le Spleen de Paris : « Je viens de voir l’image du vieil homme de lettres qui a survécu à la génération dont il fut le brillant amuseur ; du vieux poète sans amis, sans famille, sans enfants, dégradé par la misère et par l'ingratitude publique, et dans la baraque de qui le monde oublieux ne veut plus entrer » $(O C$ 1:297). Voir les pages éclairantes consacrées à cette figure par Jean Starobinski, Portrait de l'artiste en saltimbanque (Genève : A. Skira, 1970), 83-99.

${ }^{36}$ Par exemple, dans une lettre de mai 1864 à Hetzel, Baudelaire se déclare « passablement irrité pour avoir vu [s]on nom prostitué dans des livres pour lesquels [il n'a] aucun goût » (Corr 2:365).

${ }^{37}$ Littré fournit deux définitions de paranymphe qui, comme le note Pichois, paraissent combinées dans le titre du poème : « $1^{\circ}$ Terme d'antiquité grecque. Le jeune ami du marié qui, assis sur le char à côté de lui, va chercher la mariée ; $[\ldots] 2^{\circ}[\ldots]$ Il s'est dit, dans les anciennes facultés de théologie et de médecine, d'un discours solennel que l'on prononce à la fin de chaque licence et où l'orateur faisait l'éloge des licenciés ». Dictionnaire de la langue française, 3:941. La nymphe est cependant définie par Delvau comme la « Déesse qui consent à sortir de son nuage pour entrer dans le lit d'un homme qui la paie pour cela », à savoir une prostituée. Alfred Delvau, Dictionnaire érotique moderne, par un professeur de langue verte (Bâle : K. Schmidt, s.d. [Bruxelles : J. Gay, 1864 ?]), 278.

${ }^{38}$ Une « note de l'éditeur» (en fait de l'auteur) précise : « À la Messe noire. Comme ces poètes sont superstitieux ! » Le 23 janvier 1866, Baudelaire se renseigne auprès de PouletMalassis : «Le mot Cas peut-il s'appliquer au Cul comme à la pine, ou en est-il l'antipode ? Il s'agit du Diable » (Corr 2:577). D'après Delvau, le cas est « le membre viril aussi bien que la nature de la femme » (Delvau 82). On sait que la messe noire mettant en scène des rencontres charnelles entre la femme et le Diable est l'un des thèmes de prédilection de Rops. 
${ }^{39}$ Voir les lettres à Mendès de janvier 1866 (Corr 2:573-78). Le 26 janvier, Baudelaire le prévient par exemple : «La pièce intitulée Le Monstre, si toutefois vous osez l'imprimer, a maintenant quinze ou seize couplets, avec un certain air archaïque qui en sauve un peu la crudité »(Corr 2:578).

${ }^{40}$ Lettre de Mendès à Baudelaire de janvier 1866 (Crépet 397).

${ }^{41}$ Voir n. 14.

${ }^{42}$ La première édition tolérée des Épaves et des « pièces condamnées » des Fleurs du Mal date de la publication des Euvres posthumes [éd. Féli Gautier et Jacques Crépet] (Paris : Mercure de France, 1908).

${ }^{43}$ Reproduit dans Le Manuscrit autographe. Numéro spécial consacré à Charles Baudelaire (Paris : A. Blaizot, 1927), 77.

${ }^{44}$ Voir, par exemple, l'édition critique des Fleurs de Mal établie par Jacques Crépet et Georges Blin (Paris : J. Corti, 1942), 600-03 ; ainsi que celle annotée par Antoine Adam (Paris : Classiques Garnier, 1959), 454-55.

${ }^{45}$ Cette Berthe, dont on ne sait pas grand-chose, bien qu'Alfred Stevens la désigne comme la « dernière maîtresse » de Baudelaire qu'il aurait « connue », a été photographiée par Weyler et dessinée par Baudelaire au-dessus du poème « Les Yeux de Berthe » en 1864 (Pichois et Ruchon, Iconographie, $\mathrm{n}^{\mathrm{o}}$ 140-44).

${ }^{46}$ Par exemple cet autre sonnet, non daté, également adressé à Poulet-Malassis : «Mon cher, je suis venu chez vous / Pour entendre une langue humaine ; / Comme un, qui, parmi les Papous, / Chercherait son ancienne Athêne. // Puisque chez les Topinambous / Dieu me fait faire quarantaine, / Aux sots je préfère les fous /—Dont je suis, chose, hélas ! certaine [...]» (Corr 2:429-30).

${ }^{47}$ Pichois ne l'édite, on l'a vu, que dans la Correspondance du poète. 
${ }^{48}$ Voir les vers de Nicolas Pradon en Réponse à la satire [contre les femmes] de Boileau :

«Il est vray que privé des dons de la nature, / Le Ciel ne te forma que pour leur faire injure [aux femmes] », Réponse à la satire X du sieur D*** (Paris : R.-J.-B. de La Caille, 1694), 2, http://gallica.bnf.fr/ark:/12148/bpt6k5723538b. Consulté le 8 décembre 2017.

${ }^{49}$ L'ouvrage, comprenant le frontispice, est consultable en ligne :

https://books.google.com/books?id=HPruBWD6X-0C. Consulté le 8 décembre 2017.

${ }^{50}$ Alphonse Karr rappelle cette légende : «Boileau enfant et encore en jaquette fut, dit-on, renversé dans une cour par un dindon très-malfaisant, du bec duquel on ne l'arracha que fort maltraité pour le présent et pour l'avenir ». Alphonse Karr, Les Femmes (Paris : M. Lévy, 1853), 6 .

${ }^{51}$ Pour envisager une hypothèse hasardeuse, on pourrait voir dans l' « octogénaire » auquel est attribué L'Art priapique le couple Baudelaire-Poulet-Malassis, qui étaient tous deux quadragénaires en 1865.

${ }^{52}$ Voir la section «Bouffonneries » qui conclut Les Épaves, en particulier les deux quatrains du « Cabaret folâtre » qui clôt le recueil : « Vous qui raffolez des squelettes / Et des emblèmes détestés, / Pour épicer les voluptés, / (Fût-ce de simples omelettes !) // Vieux Pharaon, ô Monselet ! / Devant cette enseigne imprévue, / J'ai rêvé de vous : À la vue / Du Cimetière, Estaminet! (OC 1:177-78). 